\title{
Wet Dark Fluid Cosmological Model in Barber Self-Creation Theory of Gravitation
}

\author{
S. C. Wankhade ${ }^{1 *}$, A. S. Nimkar ${ }^{1}$, A. M. Pund ${ }^{2}$ \\ ${ }^{1}$ Department of Mathematics, Shri. Dr. R.G. Rathod Arts and Science College, Murtizapur, Dist. \\ Akola, India \\ ${ }^{2}$ Department of Mathematics, Shri Shivaji Education Society Amravati's Science College, Congress \\ Nagar, Nagpur, India
}

Received 18 April 2021, accepted in final revised form 23 June 2021

\begin{abstract}
This paper investigates a Bianchi type VIII cosmological model with wet dark fluid in Barber self-creation theory of gravitation. To get the determinate model of the Universe, we have assumed the relationship between metric coefficients $R$ and $S$. Also, the behavior of the model in the radiation Universe and the physical implications of the model are discussed in detail.
\end{abstract}

Keywords: Bianchi type VIII; Barber self-creation theory; Wet dark fluid.

๑) 2021 JSR Publications. ISSN: 2070-0237 (Print); 2070-0245 (Online). All rights reserved. doi: http://dx.doi.org/10.3329/jsr.v13i3.53064

J. Sci. Res. 13 (3), 869-878 (2021)

\section{Introduction}

Einstein's general theory of relativity has provided a sophisticated theory of gravitation. It does not account for satisfactory inertial properties of matter, i.e., Mach principle is not substantiated by general relativity. There have been some interesting attempts to generalize the general theory of gravitation by incorporating Mach's principle and other desired features lacking in the original theory. Barber [1] modified it by coupling the scalar field with the energy-momentum tensor so that the theory substantially accommodates Mach's principle. Barber proposed two self-creation theories based on two sets of general relativistic field equations involving matter and a scalar field. The first is a modified Brans-Dicke theory that Brans pointed out [2], which is unsatisfactory since it is not only in disagreement with the experiment but is inconsistent since the equivalence principle is violated. The second is a modification of general relativity to include the continuous creation of matter within the limits of observation. In this theory, the scalar field acts as a reciprocal gravitational constant $G \sim \varphi^{-1}$. It is postulated that this scalar field couples with the trace of the energy-momentum tensor.

Field equation of Barber Second Self Creation Theory can be written in the form,

\footnotetext{
*Corresponding author: sharvariwankhade006@gmail.com
} 


$$
G_{i j}-\frac{1}{2} R g_{i j}=-8 \pi \varphi^{-1} T_{i j}
$$

and the scalar field equations is defined by

$$
\varphi=\varphi_{; k}^{k}=\frac{8 \pi \lambda}{3} T \text {. }
$$

Where, $R_{i j}$ is Ricci Tensor, $g_{i j}$ is Metric Tensor, $R$ is Scalar Curvature, $\varphi$ is Scalar Field, $T_{i j}$ is the stress-energy tensor of the matter and $T$ stands for its trace. $\square \quad$ is the D'Alembertian, $\lambda$ is coupling constant to be determined from experiments.

Many authors [3-12] have investigated various aspects of Barber second self-creation theory by using various cosmological models.

The nature of the dark energy component of the Universe is one of the deepest mysteries of cosmology. The wet dark fluid (WDF) is a model for dark energy. This model is in the spirit of the generalized Chaplygin gas Gorini et al. [13], where a physically motivated equation of state is offered with properties relevant for the dark energy problem. Here the motivation stems from an empirical equation of state proposed by Tait [14] and Hayward [15] to treat water and aqueous solution.

The equation of state for WDF is

$$
p_{W D F}=\gamma\left(\rho_{W D F}-\rho *\right),
$$

where the parameters $\gamma$ and $\rho *$ are taken to be positive and we restrict ourselves to $0 \leq \gamma \leq$ 1.

We have an energy conservation equation as

$$
\dot{\rho}_{W D F}+3 H\left(p_{W D F}+\rho_{W D F}\right)=0 .
$$

From the equation of state (3) and using $3 H=\frac{\dot{V}}{V}$ in equation (4), where $V$ is the volume expansion, we get

$$
\rho_{W D F}=\frac{\gamma}{1+\gamma} \rho *+\frac{c}{V^{1+\gamma}}
$$

Where, $c$ is an integration constant.

WDF naturally includes two components, a piece that behaves like a cosmological constant as well as a piece that redshifts as a standard fluid with an equation of state

$$
p_{W D F}=\gamma \rho_{W D F} .
$$

If we take $c>0$, then this fluid will not violate the strong energy condition,

$$
p_{W D F}+\rho_{W D F} \geq 0 \text {. }
$$

Thus, we get

$$
\begin{aligned}
& \left(p_{W D F}+\rho_{W D F}\right)=(1+\gamma) \rho_{W D F}-\gamma \rho * \\
& =(1+\gamma) \frac{c}{V^{(1+\gamma)}} \geq 0 .
\end{aligned}
$$

The wet dark fluid has been used as dark energy in the homogeneous, isotropic FRW case by researchers [16-26] who have investigated various aspects of wet dark fluid

Hence, motivating the above discussions, in this paper, we have investigated Bianchi type VIII cosmological model in the presence of wet dark fluid in Barber self-creation theory. Our paper is organized as follows. In section 2, we derived the field equation in 
the presence of wet dark fluid in Barber self-creation theory. Section 3 deals with the solution of field equations. Section 4 contains the discussion of the physical parameters of the model. Section 5 is provided with the kinematical parameter of the obtained model. The last section contains some conclusions.

\section{Metric and Field Equation}

Since the present-day Universe is well-described by an isotropic and homogeneous FRW space-time, there are serious theoretical arguments about the existence of an anisotropic phase in the evolution of the Universe. The experimental data favors an anisotropic Universe, and hence it motivates us to study the Universe models with anisotropic background structure. The simplest anisotropic model of the Universe is homogeneous Bianchi type space-time. The advantages of these anisotropic models are their flat spatial sections. However, the expansion or contraction rates are direction-dependent, and they have a significant role in the description of the early phase evolution of the Universe. Hence, in the present paper, we consider the Bianchi type-VIII space-time in the form,

$$
d s^{2}=-d t^{2}+R^{2}\left(d \theta^{2}+\cosh ^{2} \theta d \varphi^{2}\right)+S^{2}(d \psi+\sinh d \varphi)^{2},
$$

Where $R$ and $S$ are functions of time $(t)$ only.

Here we have taken an attempt to build a cosmological model in this space-time with Wet Dark Fluid (WDF) having energy-momentum tensor of the form

$$
T_{i j}=\left(\rho_{W D F}+p_{W D F}\right) u_{i} u_{j}+p_{W D F} .
$$

Where $p_{W D F}$ is a pressure $\rho_{W D F}$ is the density of wet dark fluid, $u^{i}=(0,0,0,1)$ denotes the four-velocity vector in co-moving coordinates, which satisfies the condition.

From (9), we get

$$
T_{1}^{1}=T_{2}^{2}=T_{3}^{3}=p_{W D F}, \text { and } T_{4}^{4}=-\rho_{W D F} .
$$

With the help of components of energy-momentum tensor provided in equation (10), the components of field equations of Barber second self-creation theory from equation (2) are obtained as

$$
\begin{aligned}
& \frac{\ddot{R}}{R}+\frac{\ddot{S}}{S}+\frac{\dot{R} \dot{S}}{R S}+\frac{1}{4} \frac{S^{2}}{R^{4}}=-8 \pi \varphi^{-1} p_{W D F}, \\
& \frac{2 \ddot{R}}{R}+\frac{\dot{R}^{2}}{R^{2}}-\frac{1}{R^{2}}-\frac{3}{4} \frac{S^{2}}{R^{4}}=-8 \pi \varphi^{-1} p_{W D F}, \\
& \frac{2 \dot{R} \dot{S}}{R S}+\frac{\dot{R}^{2}}{R^{2}}-\frac{1}{R^{2}}-\frac{1}{4} \frac{S^{2}}{R^{4}}=8 \pi \varphi^{-1} \rho_{W D F}, \\
& \ddot{\varphi}+\left(\frac{2 \dot{R}}{R}+\frac{\dot{S}}{S}\right) \dot{\varphi}=\frac{8 \pi \lambda}{3}\left(3 p_{W D F}-\rho_{W D F}\right),
\end{aligned}
$$

Where, the overhead dots of the field variable denote ordinary differentiation with respect to time. 


\section{Solutions of Field Equations}

The set of differential equations (11) to (13) be the three independent equations with five unknown $R, S, \varphi, p_{W D F} \rho_{W D F}$ s. In order to get the deterministic solution, we have assumed the following conditions:

i) As Collins have pointed out that for spatially homogeneous metric, the normal congruence to the homogeneous expansion satisfies the condition $\frac{\sigma}{H}$ constant. The observations of the velocity-red-shift relation for extragalactic sources suggest that Hubble expansion of the Universe is isotropic today within $\approx 30$ percent, i.e., redshift studies dwelling the limit $\frac{\sigma}{H} \leq 0.3$ (constant) on the ratio of shear $\sigma$ Hubble constant $H$ in the neighborhood of our Galaxy today. It gives the relation between the metric potentials as follows,

$$
S=R^{n} \text {. }
$$

ii) We use the condition for the radiation Universe which is given by,

$$
3 p_{W D F}-\rho_{W D F}=0,
$$

With the help of equation (15) and (16), equations (11), (12), and (13) reduces to,

$$
\frac{(n+2) \ddot{R}}{R}+n(n-1) \frac{\dot{R}^{2}}{R}=0 .
$$

Equation (17) gives the solution as follows,

$$
\begin{aligned}
& R=\left(k_{3} t+k_{4}\right)^{N}, \\
& S=\left(k_{3} t+k_{4}\right)^{N_{1}} .
\end{aligned}
$$

And the scalar field is given by,

$$
\varphi=\frac{k_{7}}{\left(k_{3} t+k_{4}\right)^{N_{3}}}+k_{6},
$$

Where, $N=\frac{n+2}{n^{2}+2} ; n \neq \sqrt{-2}, N_{1}=n N$ and $N_{3}=\frac{2(2 n+1)}{n^{2}+2}, n \neq \sqrt{-2}$.

Using equations (18) and (19), the cosmological model provided in equation (8) takes the form,

$d s^{2}=-d t^{2}+\left(k_{3} t+k_{4}\right)^{2 N}\left(d \theta^{2}+\cosh ^{2} \theta d \varphi^{2}\right)+\left(k_{3} t+k_{4}\right)^{2 N_{1}}\left(d \psi+\sinh ^{2} \theta d \varphi\right)^{2}$.

From the above model (21), it is observed that the metric potentials are different; hence it represents an anisotropic model but for the constant $n$ if $n=1$, these are identical, which represents an isotropic model. Also, at $t=0$, the model is constant, but at a specific time $t=t_{s}=\frac{k_{4}}{k_{3}}$, the matric potential in the model vanishes; hence the model represents a singular model. Also, there is no such relation between the constants in the model for which the model shows isotropy. 


\section{Physical Parameters}

With the help of equations (18), (19), and (20), the physical parameters of the model such as pressure, energy density, and equation of state parameter of wet dark fluid are obtained as follows,

The pressure of wet dark fluid is given by,

$$
p_{W D F}=\frac{1}{24 \pi}\left(\frac{k_{7}}{\left(k_{3} t+k_{4}\right)^{N_{3}}}+k_{6}\right)\left\{\frac{(2 n+1) N^{2} k_{3}{ }^{2}}{\left(k_{3} t+k_{4}\right)^{2}}-\frac{1}{\left(k_{3} t+k_{4}\right)^{2 N}}-\frac{1}{4}\left(k_{3} t+k_{4}\right)^{2 N(n-2)}\right\} .
$$

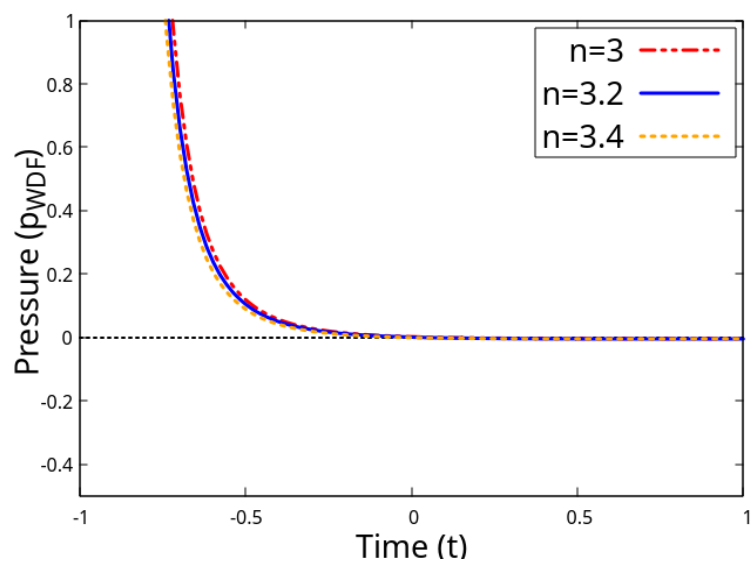

Fig. 1. Behavior of pressure of wet dark fluid versus time with the appropriate choice of constants.

The energy density of wet dark fluid is given by,

$$
\rho_{W D F}=\frac{1}{8 \pi}\left(\frac{k_{7}}{\left(k_{3} t+k_{4}\right)^{N_{3}}}+k_{6}\right)\left\{\frac{(2 n+1) N^{2} k_{3}^{2}}{\left(k_{3} t+k_{4}\right)^{2}}-\frac{1}{\left(k_{3} t+k_{4}\right)^{2 N}}-\frac{1}{4}\left(k_{3} t+k_{4}\right)^{2 N(n-2)}\right\} .
$$

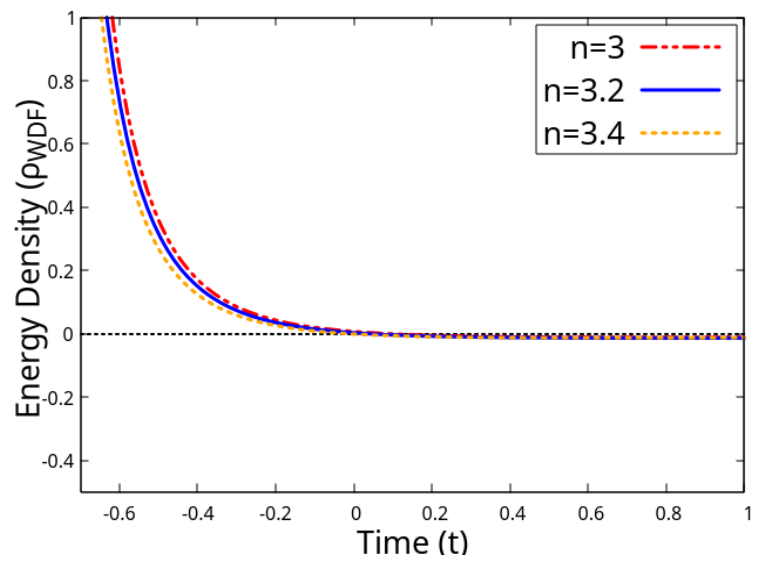

Fig. 2. Behavior of energy density of wet dark fluid versus time with the appropriate choice of constants. 


\section{Wet Dark Fluid Cosmological Model}

Equation of state Parameter,

$$
\omega=\frac{p_{W D F}}{\rho_{W D F}}=\frac{1}{3}
$$

\section{Kinematical Parameters}

The kinematical parameters such as the spatial volume, Hubble's parameter, expansion scalar, the anisotropic parameter, and shear scalar of the model (21), which are of cosmological importance, are respectively given by Spatial volume,

$$
V=\sqrt{-g}=\left(k_{3} t+k_{4}\right)^{\frac{(n+2)^{2}}{n^{2}+2}} \cosh \theta .
$$

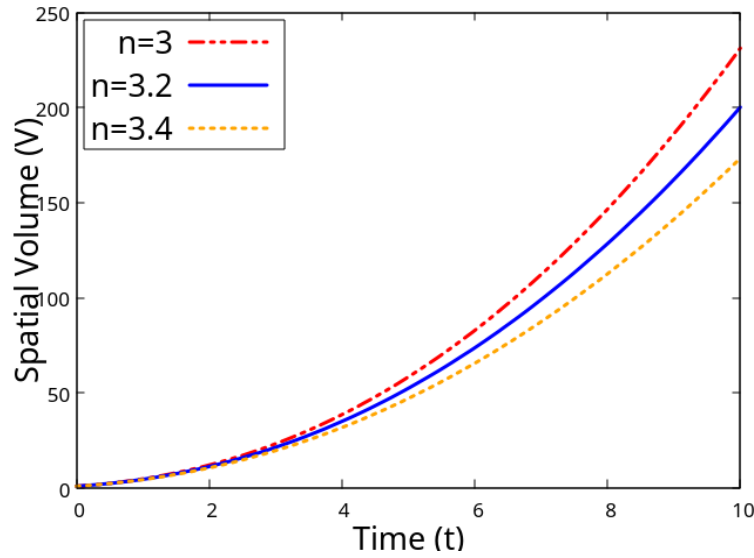

Fig. 3. Behavior of spatial volume of the model versus time with the appropriate choice of constants.

Equation (25) represents the spatial volume of the model, which is also depicted in Fig. 3. From Fig., it is observed that the spatial volume of the model is constant at an initial timet $=0$ and expands exponentially as $t$ increases and becomes infinitely large at $t$ $=\infty$. Also, at the specific timet $=t_{s}=\frac{d}{c}$ the spatial volume of the model is zero; hence the model represents a singular model. At the singular point $t_{s}$ for $t_{4}>0$ the volume of the Universe vanish. Thus we can conclude that from the observation, the Universe starts its expansion from zero volume at a singular point.

Expansion Scalar,

$$
\theta=\frac{(n+2)^{2}}{\left(n^{2}+2\right)} \frac{k_{3}}{k_{3} t+k_{4}} .
$$




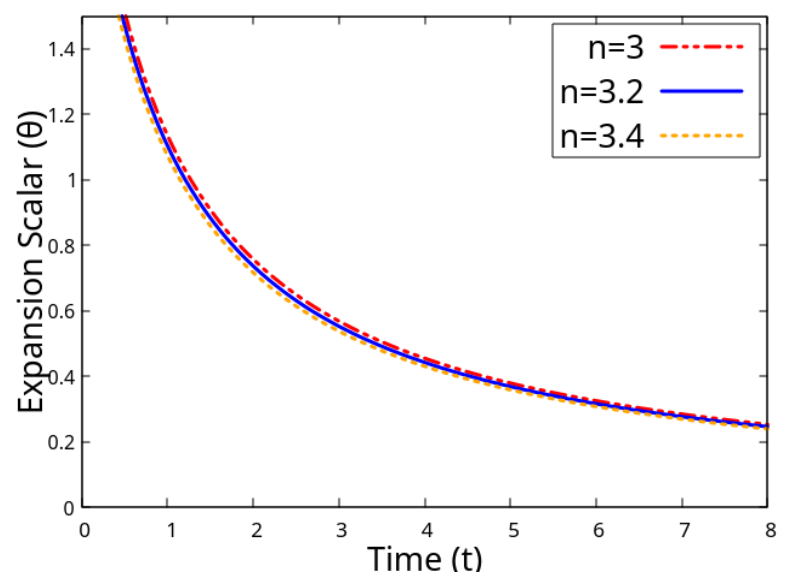

Fig. 4. Behavior of expansion scalar of the model versus time with the appropriate choice of constants.

Hubble Parameter,

$$
H=\frac{1}{3} \frac{(n+2)^{2}}{\left(n^{2}+2\right)} \frac{k_{3}}{k_{3} t+k_{4}} .
$$

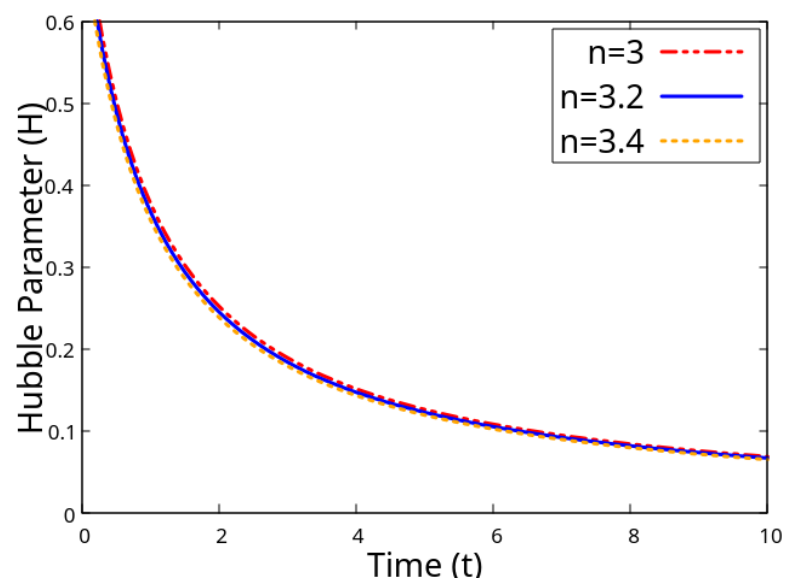

Fig. 5. Behavior of Hubble's parameter of the model versus time with the appropriate choice of constants.

From Figs. 4 and 5, it is observed that the expansion scalar and Hubble's parameter both are the decreasing functions of time while the Hubble's parameter, expansion scalar, both are infinitely large at a singular point $t_{s}$.

Shear Scalar,

$$
\sigma^{2}=\frac{1}{2} \sigma_{i j} \sigma_{i j}=\frac{(n+2)^{4}}{6\left(n^{2}+2\right)^{2}} \frac{k_{3}^{2}}{\left(k_{3} t+k_{4}\right)^{2}} .
$$




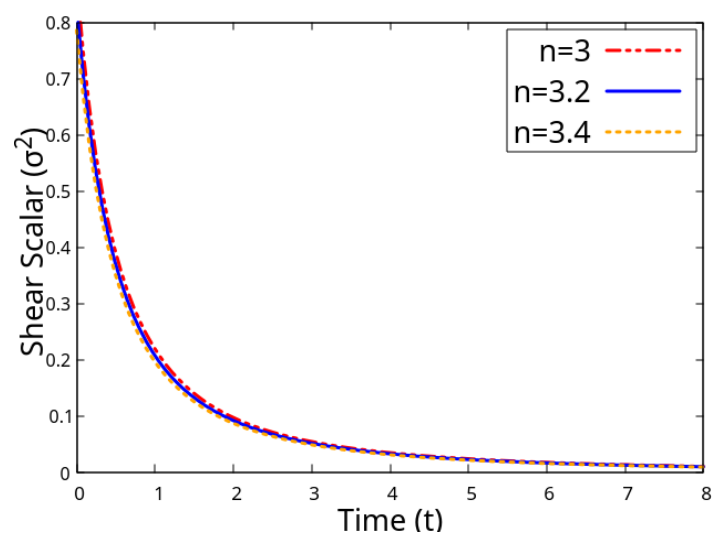

Fig. 6. Behavior of shear scalar of the model versus time with the appropriate choice of constants.

Since, at a singular point, we obtained $\sigma^{2}=0$, which implies that our Universe is homogeneous, isotropic and shear-free. The Universe is expanding with the increase of cosmic time, but the rate of expansion decreases to a constant value, showing that the Universe starts evolving with zero volume at $t \rightarrow 0$, an infinite rate of expansion. Anisotropy Parameter,

$$
\Delta=\frac{1}{3} \sum_{i=1}^{3}\left(\frac{H_{i}-H}{H}\right)^{2}=3\left(\frac{n-1}{n+2}\right)^{2}
$$

Where, directional Hubble parameter is given by,

$$
H_{1}=\frac{n+2}{n^{2}+2} \frac{k_{3}}{k_{3} t+k_{4}}, H_{2}=H_{3}=\frac{n(n+2)}{n^{2}+2} \frac{k_{3}}{k_{3} t+k_{4}}
$$

Average Scale factor,

$$
a(t)=\left(k_{3} t+k_{4}\right)^{\frac{(n+2)^{2}}{3\left(n^{2}+2\right)}} \cosh ^{\frac{1}{3}} \theta
$$

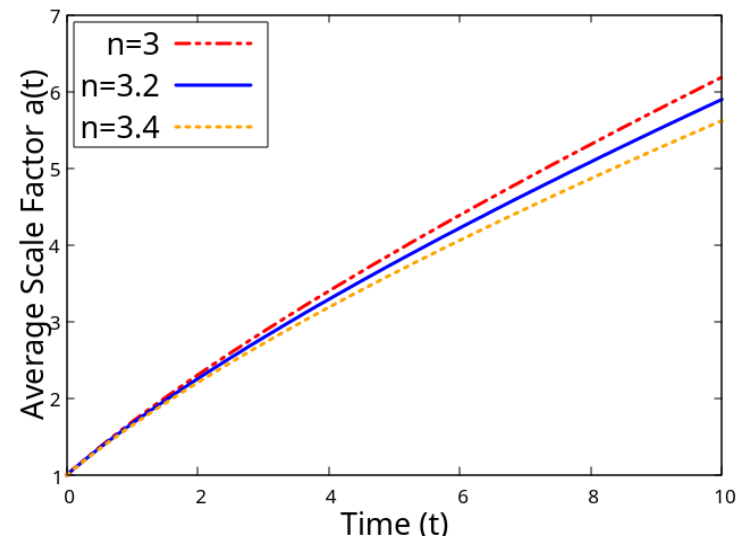

Fig. 7. Behavior of average scale factor of the model versus time with the appropriate choice of constants. 
Deceleration Parameter,

$$
q=2\left(\frac{n-1}{n+2}\right)^{2}
$$

Decomposition of time like tidal tensor is

$$
\begin{aligned}
& u_{a ; b}=-k_{3} N\left\{\left(k_{3} t+k_{4}\right)^{2 N-1}\left[1+\cosh ^{2} \theta\right]+n\left(k_{3} t+k_{4}\right)^{2 n N-1}\right\} \\
& \text { Vorticity } \omega_{11}=\omega_{22}=\omega_{33}=\omega_{44}=0 .
\end{aligned}
$$

Redshift,

For redshift, the scale factor $a\left(t_{z}\right)$ of the Universe is related to $a_{0}$ by

$$
1+z=\frac{a_{0}}{a}
$$

Where, subscript 0 denotes the present phase. $a_{0}$ is the present scale factor.

From equation (30), we get

$$
z=-1+\left(\frac{k_{3} t_{0}+k_{4}}{k_{3} t+k_{4}}\right)^{\frac{(n+2)^{2}}{3\left(n^{2}+2\right)}}
$$

Here, $t_{0}$ is the age of the Universe at the present time $(z=0)$.

\section{Conclusion}

In the analysis of wet dark fluid cosmological model in Barber second self-creation theory of gravitation, the exact solution of the field equations is obtained by solving them with the help of a relation between the metric potentials. It is observed that the spatial volume is constant $t \rightarrow 0$ while the Universe starts its expansion from zero volume at a singular point. Therefore, the model starts evolving with constant volume $t=0$ and expands with cosmic time along with other parameters such as expansion scalar, shear scalar and Hubble's parameter are constants, but at a singular point $t_{s}$, the spatial volume vanishes, and other parameters diverge. Hence derived model has a Big-bang type of singularity at the singular point $t_{s}$.

\section{References}

1. G. A. Barber, Gen. Relat. Gravit. 14, 117 (1982). https://doi.org/10.1007/BF00756918

2. C. Brans and R. H. Dicke, Phys. Rev. 124, 925 (1961). https://doi.org/10.1103/PhysRev.124.925

3. L. O. Pimentel, Astrophys. Space Sci. 116, 395 (1985).

4. L. O. Pimentel, Astrophys. Space Sci. 139, 13 (1987). https://doi.org/10.1007/BF00643809

5. Venkateswarlu and D. R. K. Reddy, Astrophys Space Sci. 151, 353 (1989). https://doi.org/10.1007/BF00640501

6. Shanti, K., Rao, V.U.M.: Astrophys. Space Sci. 179, 147 (1991). https://doi.org/10.1007/BF00642359

7. G. Mohanty, B. Mishra, and A. K. Biswal, Czechoslovak J. Phys. 52, 1289 (2002). https://doi.org/10.1023/A:1021854925919

8. G. Mohanty, Astrophys. Space Sci. 281, 633 (2002). https://doi.org/10.1023/A:1015858621340

9. K. S. Adhav, A. S. Nimkar, and M. V. Dawande, Int. J. Theor. Phys. 47, 3201 (2008). https://doi.org/10.1007/s10773-008-9755-5 
10. S. D. Katore, A. Y. Shaikh, and S. A. Bhaskar, Sci. Revs. Chem. Commun. 2, 463 (2012).

11. V. R. Chirde and S. H. Shekh, African Rev. Phys. 9, 399 (2014).

12. D. D. Pawar and Y. S. Solanke, Adv. Higher Energy Phys. 2014, ID 859638 (2014). https://doi.org/10.1155/2014/859638

13. V. Gorini, A. Kamenshchik, U. Moschella, and V. Pasquier, arXiv:gr-qc/0403062 (2004).

14. P. G.Tait, Voyage of HMS Challanger, 2, 1 (1988).

15. A. T. J. Hayward, Brit. J. Appl. Phys. 18, 965 (1967). https://doi.org/10.1088/0508-3443/18/7/312

16. R. Holman and S. Naidu, arXiv:astro-ph/0408102 (2005).

17. R. Chaubey, Int. J. Astrono. Astrophys. 1 (2011).

18. K. S. Adhav, V. G. Mete, R. S. Thakare, and A. M. Pund, Int. J. Theor. Phys. 50, 164 (2011). https://doi.org/10.1007/s10773-010-0504-1

19. B. Mishra and P. K. Sahoo, Astrophys. Space Sci. 343, 491 (2014). https://doi.org/10.1007/s10509-013-1652-6

20. P. K. Sahoo and B. Mishra, Canadian J. Phys. 92, 9 (2014). https://doi.org/10.1139/cip-2014-0348

21. G. S. Samanta, S. Jaiswal, and S. K. Biswal, Eur. Phys. J. Plus 129, 48 (2014). https://doi.org/10.1140/epjp/i2014-14048-8

22. A. S. Nimkar and A. M. Pund, IOSR J. Math. 11, 47 (2015).

23. V. R. Chirde and V. P. Kadam, Int. J. Innovat. Res. Sci. Technol. 3, 5 (2016).

24. S. D. Deo, G. S. Punwatkar, and U. M. Patil, Int. J. Math. Archive, 7, 3 (2016).

25. S. Angit, R. Rakesh, and R. Chaubey, Int. J. Geometric Methods in Modern Phys. 16, ID 1950124 (2019). https://doi.org/10.1142/S021988781950124X

26. V. J. Dagwal, Canadian J. Phys. 98 (2020). https://doi.org/10.1139/cjp-2019-0226 